\title{
Effect of Trp-P-1 on the Growth of Cultured Rat Astrocytes
}

\author{
Mihoko TABUCHI, Jin-ichi ITO', Tetsuo ISHIDA and Akiyoshi HOSONO² \\ Yonezawa Women's College of Yamagata Prefecture, Yonezawa-shi 992-0025, Japan \\ ${ }^{1}$ School of Medicine, Nagoya City. University, Mizuho-ku, Nagoya-shi 467-0002, Japan \\ ${ }^{2}$ Faculty of Agriculture, Shinshu University, Minamiminowa-mura, Nagano-ken 399-4598, Japan
}

(Received December 6, 1999 ; Accepted February 18, 2000)

\begin{abstract}
The effects of Trp-P-1 (3-amino-1,4-dimethyl-5H-pyrido[4,3-b]indole) on the growth of astrocytes were investigated by in vitroculture experiments supplemented with Trp-P-1. Cultured astrocytes were prepared from the brains of 18 days old fetuses of Wistar rats and were incubated in F-10 medium supplemented with fetal calf serum. The growth of astrocytes $\left(9 \times 10^{4}\right.$ cells $)$ cultured with $10 \mu \mathrm{M}$ Trp-P.1 was inhibited, and the numbers of astrocytes were significantly less than the control (in distilled water) at each time interval $(\mathrm{P}<0.01, \mathrm{P}<0.05)$. When astrocytes were cultured with varying concentrations of Trp-P-1 for 7 days, the cell number decreased in proportion to the increased dose of Trp-P-1. These results indicated that Trp-P-1 can significantly inhibit the astrocytes development in in vitro culture and the inhibition was dose dependent.
\end{abstract}

Key words : Trp-P-1, Astrocyte, Growth, Rat fetus

Animal Science Journal 71 (3) : 305-307, 2000

Daily foods contain a number of mutagenic heterocyclic amines. Among these, Trp-P-1 (3 - amino-1, 4 -dimethyl - $5 H$-pyrido $[4,3-b]$ indole) was found in the charred surface of meat and fish, which is reported to be highly mutagenic in Ames' Salmonella assay and carcinogenic to rats and mice on longterm dietary exposure ${ }^{8)}$. Therefore, the effects of antimutagenicity and safety against heterocyclic amines are well documented, especially on $\operatorname{Tr} \mathrm{p}-\mathrm{P}-1^{3)}$.

We have recently reported that administrations of Trp-P-1 to pregnant rats affected the brain weight gain by the fetuses" This led us to look into the cells or area of brain affected by Trp-P-1, thus we studied the effect of Trp-P-1 on the growth of cultured rat astrocytes, belonging to glial cells. In adult mammal, the central nervous system (CNS) consists of neurons which transmit stimulation and glial cells which are present about ten times as much as neurons. Astrocyte is typical glial cell and plays an important role in the CNS, such as neurotic structural support, metabolism of neurotransmitter and production of neurotrophic factors.

To our knowledge, there is no published report on the effect of Trp-P-1, on the growth of astrocytes in vitro, including glial cells. The present experiment was conducted to study the effect of heterocyclic amines on the growth of fetal brain.

\section{Materials and Methods}

Rat astrocytes were cultured as described by Ito and Kato ${ }^{6}$. Briefly, the brains of 18 days old Wistar rat fetuses, from which blood and meninges were removed, minced with scissors, washed three times in Tyrode's solution and treated with $0.1 \%$ trypsin in Tyrode's solution. Tyrode's solution consisted of $8 \% \mathrm{NaCl}, 0.2 \%$ $\mathrm{KCl}, 0.05 \% \quad \mathrm{NaH}_{2} \mathrm{PO}_{4}, 1 \%$ glucose and $1 \%$ $\mathrm{NaHCO}_{3}$ (all in wt/vol). The trypsin solution was removed by centrifugation $(1,500 \times g, 5$ min) and pellets were cultured in F-10 medium (Gibco, USA) supplemented with $15 \%$ fetal calf serum (FCS, Gibco) for 7 days at 37 ${ }^{\circ} \mathrm{C}$ in a humidified atmosphere of $5 \% \mathrm{CO}_{2} /$ air. Subsequently the cells were collected by treatment with $0.1 \%$ trypsin and secondary monolayer cultures were established in 6 well plates (Iwaki, Japan) using F-10 medium supplemented with $10 \% \mathrm{FCS}$. These cultures were maintained for $48 \mathrm{~h}$ at $37^{\circ} \mathrm{C}$ in a

Corresponding: Mihoko TABUCHI (fax: $+81(0) 238-22-7333$, e-mail: tabuchi@yone.ac.jp) 


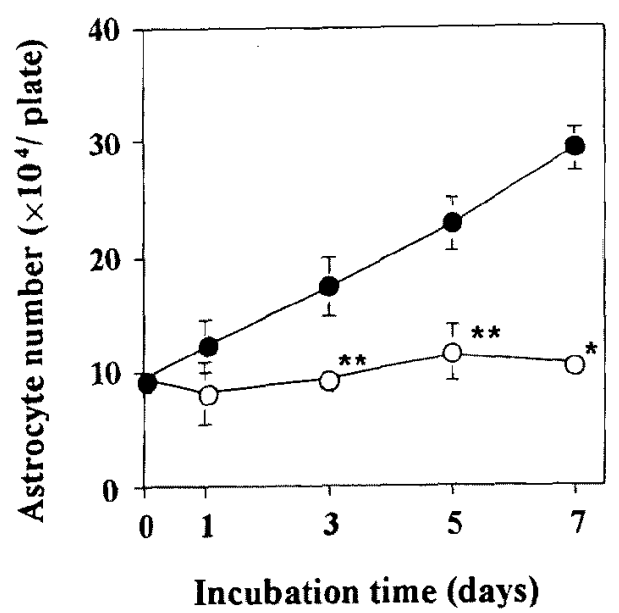

Fig. $I$ Effect of Trp-P-1 on rat astrocytes growth. Rat astrocytes were cultured with ( $O$ ) and without (O; control) $10 \mu \mathrm{M}$ Trp-P-1 at 37 ${ }^{\circ} \mathrm{C}$. Each point is the mean of the change in astrocytes number; the vertical bars represent standard errors. Asterisks indicate the level of significant difference from the control at each time interval $* \mathrm{P}<0,01, * * \mathrm{P}<0.05$.

humidified atmosphere of $5 \% \mathrm{CO}_{2} /$ air.

After $48 \mathrm{~h}$ in culture, the medium was refreshed and Trp-P-1 (Wako, Japan) dissolved distilled water was added to the fresh medium at $10 \mu \mathrm{M}$ per plate. The cells were maintained at $37^{\circ} \mathrm{C}$ in a humidified atmosphere of $5 \% \mathrm{CO}_{2} /$ air and the cells counts were taken at $1,3,5$ and 7 days after the Trp-P-1 was added. Dose dependent effect of Trp-P - 1 was also measured at $1,5,10,15$ or $20 \mu \mathrm{M}$ after 7 days in culture. The medium was refreshed for a day during incubation. The cells counts were performed in Thoma Haemacytometer (Kayagaki Irika Kogyo, Japan).

Each experiment was done three times, and the results were expressed as mean \pm SEM. Statistical difference between control and treatment values was analysed by student's $t$ test.

\section{Results and Discussion}

We have recently reported that brain weight of fetus was signilicantly decreased by the administration of Trp-P-1 to rats on the 15-17 days of pregnancy ${ }^{4}$. Further, Trp-P-1 was detected in all brain samples exposed to TrPP-1 by HPLC analysis. These results suggested the possibility of Trp-P-1 passing the placental and blood-brain bartier, and may

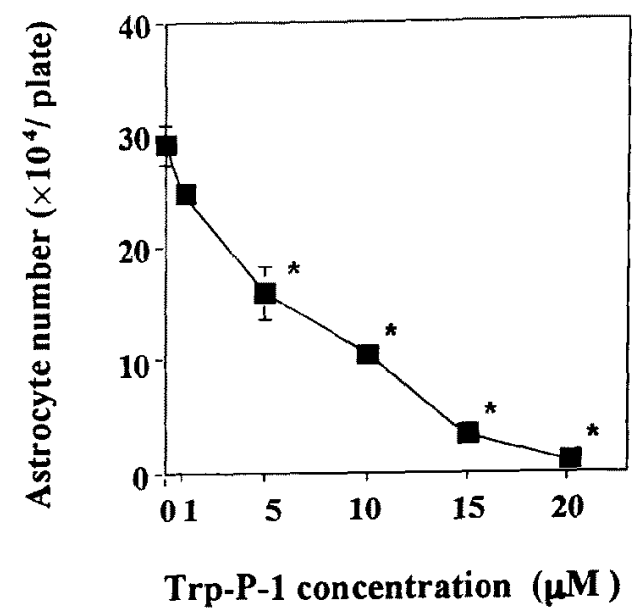

Fig. 2 Effect of different concentration of Trp-P-1 on rat astrocytes growth. Rat astrocytes were cultured with varying concentrations of Trp-P- 1 at $37^{\circ} \mathrm{C}$ for 7 days. Each point is the mean of the change in astrocytes number; the vertical bars represent standard errors. Asterisks indicate the level of significant difference in the absence of Trp-p-1 at each concentrations $* \mathrm{P}<0.01$.

damage the fetal brain.

In present study, the effects of Trp-P-1 on the growth of cultured astrocytes prepared from the brains of rat fetuses were examined. The growth of astrocytes $\left(9 \times 10^{4}\right.$ cells $)$ cultured with $10 \mu \mathrm{M}$ Trp-P-1 are shown in Fig. 1. Astrocytes treated with distilled water (control) instead of Trp-P-1 solution increased linearly over time. After 7 days incubation, the number of astrocytes was about three times as much as when it was started and the cells in plates were saturated. In contrast, the astrocytes cultured with Trp-P-1 were inhibited and the cell number was significantly $(\mathrm{P}<0.05, \mathrm{P}<0.01)$ different from the control, at 3,5 and 7 days. The growth of astrocytes $\left(9 \times 10^{4}\right.$ cells $)$ cultured with varying concentration of Trp-P-1 are shown in Fig.2. The numbers of astrocytes were decreased in proportion to the increased dose of Trp-P-1. When astrocytes were cultured with 5-20uM Trp-P-1, the cells numbers were significantly less than without Trp-Pl $(P<0.01)$. In presence of $10 \mu \mathrm{M} \operatorname{Trp}-\mathrm{P}-1$, the number of astrocytes was about $9 \times 10^{4}$ cells, which was as much as before the Trp-P.l treatment. However, the numbers of astrocytes cultured in medium supplemented with 1 or $5 \mu \mathrm{M}$ Trp-P-1 were less than the control and were more than 9 $\times 10^{4}$ cells. When these astrocytes were 
incubated for more than 7 days, the culture plates were saturated with alls grown (data not shown). On the other hand, astrocytes exposed to 15 or $20 \mu \mathrm{M}$ Trp-P-1 were not at all developed, possibly due to the cell death occurred at higher concentration of TrpP-1. These results indicated that lower dose of Trp-P-1 might function as a growth inhibitor of astrocytes and as a toxin at higher dose.

Davis et $a l .{ }^{23}$ reported that food mutagen IQ (2-amino-3-methylimidazo $[4,5-f]$ quinoline) and PhIP (2-amino-1-methyl-6-phenylimidazo $[4,5-b]$ pyridine), which are heterocyclic amines, and their metabolites were toxic to cardiomyocytes and DNA adducts formation were observed both in vitro and in vivo in rats. Moreover, it has been demonstrated that TrpP-1 is toxic to cells by forming DNAadducts ${ }^{10)}$ In addition to these facts, the formation of DNA adduct by Trp-P-1 may play an important role in the inhibition of astrocytes growth. However, further study especially, morphological study by using light and electron microscope needs to clarify the mechanism of astrocyte damage by Trp-P-1

Trp-P.1 was widely found not only in cooked foods but also in dirty air, rainwater and cigarette smoke and was also detected in human plasma, urine and bile acid ${ }^{7)}$. Further, it is reported that the calculated average dietary intake of heterocyclic amines is about 0.4 $1.6 \mu \mathrm{g} / \mathrm{day} /$ individua $1^{9}$ and it accumulates in different parts of the body. These reports indicated that individuals who consume cooked food as part of their daily diet are continuously exposed to heterocyclic amines and cannot avert. Recently, Trp-P-1 was also found to function as a neurotoxin ${ }^{5}$. It was transported into the brain through the blood-brain barrier of mice ${ }^{1)}$.

The CNS consists of neuron, astrocyte or other glial cells and they are complexly implicated. In addition to the fact which the administrations of Trp-P-1 showed decrease in brain weight of fetal rats, our result indicated that low concentration of Trp-P-1 may play a role in inhibiting the development of CNS of fetus.

\section{References}

1) Brand I, Gustafsson J, Rafter J. Distribution of carcinogenic tryptophan pyrolysis product Trp-P-1 in control, 9-hydroxyellipticine and b- naphthoflavone pretreated mice. Carcinogenesis, 4: 1291-1296. 1983.

2) Davis CD, Farb A, Thorgeirsson SS, Virmani R, Synderwine EG. Cardiotoxicity of heterocyclic amine food mutagens in cultured myocyte in rats. Toxicology and Applied Pharmacology, 124: 201-211. 1994.

3) Hosono A, Usman, Ohba R. Inhibitory activity of maillard reaction products against Trp-P-1-induced mutagenicity of Salmonella typhimurium TA98 streptomycin-dependent strain assayed in the absence of S-9mix. Bioscience, Biotechnology, and Biochemistry, 61: 424-426. 1997.

4) Hosono A, Tabuchi M, Ito J. Effect of administration of lactic acid bacteria to pregnant rats in detoxifying Trp-P-I and brain weight gain by the fetus. Aminal Science and Technology, 69: 1050-1054. 1998.

5) Ichinose $\mathrm{H}$, Ozaki $\mathrm{N}$, Nakahara D, Naoi $\mathrm{M}$, Wakabayashi K, Sugimura T, Nagatsu T. Effect of heterocyclic amines in food on dopamine metabolism in nigro-striatal dopaminergic neurons. Biochemistry and Pharmacology, 37: 3289-3295. 1988.

6) Ito J, Kato T. Culture of astrocyte. In: Manual of Neuron Biochemistry. 143-150. Youdo Company. Tokyo. 1992.

7) Manabe S, Wada O. Analysis of human plasma as an exposure level monitor for carcinogenic tryptophan pyrolysis products. Mutation Research, 209: 33-38. 1988 .

8) Meester CD. Bacterial mutagenicity of heterocyclic amines found in heat-processed food. Mutation Research, 221: 235- 262. 1989.

9) Wakabayashi K, Nagano M, Esumi H, Sugimura T. Food-derived mutagens and carcinogens. Cancer Research, 52: 2092 2098. 1992.

10) Yamashita K, Umemoto A, Grivas S, Kato $\mathrm{S}$, Sugimura $\mathrm{T}$. In vitro reaction of hydroxyamino derivatives of MeIQx, GluP-1 and Trp-P-1 with DNA: $32 \mathrm{P}$-postlabeling analys is of DNA adducts formed in vivo by the parent amines and in vitro by their hydroxyamino derivatives. Mutagenesis, 3: 515-520, 1988. 